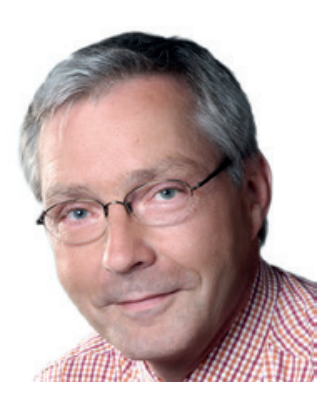

\title{
Positive Influence of Probiotics on the Gut-Skin Axis
}

\author{
Michael Sticherling
}

Hautklinik, Universitätsklinikum Erlangen, Erlangen, Germany

Abstract from Szántó M, Dózsa A, Antal D, et al.: Targeting the gut-skin axis - probiotics as new tools for skin disorder management? Experimental Dermatology. 2019;28:1210-1218.

\section{Keywords}

Atopic dermatitis · Inflammatory skin diseases · Pathogenesis . Psoriasis

\begin{abstract}
The existence of a gut-skin axis is supported by increasing evidence, but its translational potential is not widely recognized. Studies linked inflammatory skin diseases to an imbalanced gut microbiome; hence, the modulation of the gut microbiota to improve skin condition seems to be a feasible approach. Today, there is a growing interest in natural products as alternatives to synthetic drugs. In this respect, oral probiotics could be a simple, safe and cheap modality in the therapeutic management of skin inflammation. Unfortunately, very few studies have looked into how probiotic supplementation influences inflammatory skin disor-
\end{abstract}

ders. The results, though promising, are difficult to implement in clinical practice due to the heterogeneity of the applied supplemental regimen in the different studies. In this Viewpoint, we aim to encourage the conduction of more research in that direction to explore unambiguously the therapeutic potential of oral probiotics in dermatology. We focus on the most common inflammatory skin diseases (atopic dermatitis, psoriasis, rosacea, acne vulgaris) with an associated gut dysbiosis, but we also discuss some less common, but very serious skin pathologies (eg erythema nodosum, pyoderma gangrenosum, hidradenitis suppurativa) that are possibly linked to a disturbed gut flora composition. We dissect the possible mechanisms along the gut-skin axis and highlight novel points where probiotics could interfere in this communication in the diseased state.

(c) 2019 John Wiley \& Sons A/S 


\section{Knowledge Transfer}

\section{Background}

Links between the condition of the skin/skin diseases and gastrointestinal diseases have long since been established in empirical medicine. Apart from an apparent influence on the skin by various internal diseases, abnormal colonisation of the gut without clinically apparent symptoms is suspected in cases of urticaria and rosacea, for example, but also in other chronic inflammatory skin diseases. In recent years, research on the microbiome, that is, the entirety of the benign microbes colonising an organ, has significantly increased in this respect and it has been able to demonstrate important links between a colonisation of the skin and gut for their own integrity in each case but also for other organs in the human body.

\section{Probiotics in Cases of Inflammatory Skin Diseases}

The degree to which the probiotics used to date in alternative medicine can be used is described by Szántó et al. in their article cited in «Viewpoint» as an example for atopic dermatitis (AD), psoriasis and acne vulgaris. Probiotics are living microorganisms which, taken orally, lead to an improvement in health. Prebiotics, by contrast, are food components which are fermented by or promote the growth of gut microbes. Bacterial strains of probiotics, such as certain lactobacilli, are able to increase anti-inflammatory cytokines such as IL-10, and this in turn induces peripheral regulatory T-cells as well as the release of various hypothalamic hormones. The current pathogenetic understanding thus views the immune system of the gut as what is referred to as «gut-associated lymphoid tissue» (GALT) as well as the microbiome and the entirety of the bioactive metabolites produced by it, referred to as the metabolome, as important components of a gut-skin axis.

\section{Atopic Dermatitis and Psoriasis}

In the case of $A D$, the topical application of probiotic bacterial strains can already lead to an improvement. The influence of a vaginal delivery versus a Caesarean section with regard to the microbial colonisation of the child is especially significant for AD. The pre- and postnatal probiotic supplementation of children could possibly also reduce the risk of developing AD. Various bacterial metabolites such as free phenols and paracresol can disrupt the epidermal barrier and thus promote the development of the skin disease. Indeed, increased intestinal permeability has been described in the case of AD as compared to healthy persons. The mutual influence of vitamin $D$ and probiotics is also interesting, given the concomitant identification of decreased vitamin D levels in correlation to the severity of AD and also of psoriasis. In the latter case, the significance of short-chain fatty acids which arise from intestinal bacteria through fermentation was able to be demonstrated in the regulation of Th-17 cells such that there may be a connection here as well between the gut and the skin.

\section{Acne Vulgaris and Others}

Apart from an abnormal colonisation of the skin in the case of acne vulgaris as a topic which has been investigated for a long time, diets and - recently proven - the gut microbiome are also important. In patients, the intestinal microorganisms can induce the insulin-like growth factor IGF-1 and produce lipopolysaccharides, which are both pathogenetically involved. A study was in fact able to demonstrate the reduction in IGF-1 and an improvement in the clinical condition when the probiotic Lactobacillus rhamnosus SP1 was taken for 12 weeks.

The extent to which other skin diseases, such as hidradenitis suppurativa, erythema nodosum and pyoderma gangraenosum, which are frequently associated with chronic inflammatory bowel diseases, are mediated via a disrupted gut microbiome and in which probiotics play an important role remains to be investigated.

\section{Conclusion for Clinical Practice}

Overall, the disruption in homeostasis in gut flora is significant not only for the gut itself but evidently also for other organs, such as the skin. Whether topical probiotics or a combination of oral and topical probiotics are effective must be demonstrated in controlled clinical studies with clearly defined inclusion and exclusion criteria as well as clinical endpoints.

\section{Disclosure Statement}

I hereby declare that there are no conflicts of interest with regard to this commentary.

\section{Republication}

This article was first published in Karger Kompass Autoimmun 2020; 2:106-107.

Correspondence: Prof. Dr. Michael Sticherling, Hautklinik, Universitätsklinikum Erlangen, Ulmenweg 18, 91054 Erlangen, Germany, michael. sticherling@uk-erlangen 